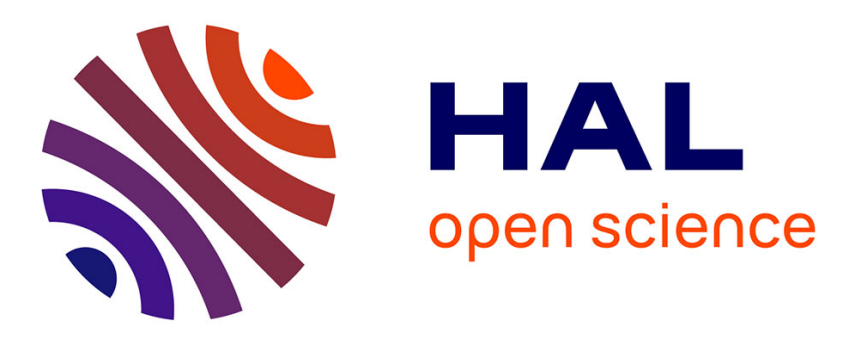

\title{
Free-volume structure of glass-As2Se3/PVP nanocomposites prepared by mechanochemical milling
}

\author{
O. Shpotyuk, A. Ingram, Z. Bujňáková, P. Baláž, Y. Shpotyuk, C. \\ Boussard-Plédel, B. Bureau
}

\section{- To cite this version:}

O. Shpotyuk, A. Ingram, Z. Bujňáková, P. Baláž, Y. Shpotyuk, et al.. Free-volume structure of glassAs2Se3/PVP nanocomposites prepared by mechanochemical milling. 9th International Conference on Times of Polymers and Composites From Aerospace to Nanotechnology, Jun 2018, Ischia, Italy. pp.020150, 10.1063/1.5046012 . hal-01874719

HAL Id: hal-01874719

https://hal-univ-rennes1.archives-ouvertes.fr/hal-01874719

Submitted on 18 Sep 2018

HAL is a multi-disciplinary open access archive for the deposit and dissemination of scientific research documents, whether they are published or not. The documents may come from teaching and research institutions in France or abroad, or from public or private research centers.
L'archive ouverte pluridisciplinaire HAL, est destinée au dépôt et à la diffusion de documents scientifiques de niveau recherche, publiés ou non, émanant des établissements d'enseignement et de recherche français ou étrangers, des laboratoires publics ou privés. 


\title{
Free-volume Structure of glass-As $\mathrm{Se}_{3} / \mathrm{PVP}$ Nanocomposites Prepared by Mechanochemical Milling
}

\author{
Oleh Shpotyuk ${ }^{\mathrm{a}, \mathrm{b}}$, Adam Ingram ${ }^{\mathrm{c}}$, Zdenka Bujňákovád, Peter Balážd, \\ Yaroslav Shpotyuk ${ }^{\mathrm{e}, \mathrm{f}}$, Catherine Boussard-Pledel ${ }^{\mathrm{g}}$, and Bruno Bureau ${ }^{\mathrm{g}}$ \\ ${ }^{a}$ Jan Dlugosz University in Czestochowa, 13/15, al. Armii Krajowej, Czestochowa, 42200, Poland \\ ${ }^{b}$ Vlokh Institute of Physical Optics, 23, Dragomanov str., Lviv, 79005, Ukraine \\ 'Opole University of Technology, 75, Ozimska str., Opole, 45370, Poland \\ ${ }^{d}$ Institute of Geotechnics of Slovak Academy of Sciences, 45, Watsonova str., Košice, 04001, Slovakia \\ ${ }^{e}$ University of Rzeszow, 1, Pigonia str., 35-310 Rzeszow, Poland \\ ${ }^{f}$ Ivan Franko National University of Lviv, 1, Universytetska str., 79000, Lviv, Ukraine \\ ${ }^{g}$ Laboratoire Verres et Céramiques UMR-CNRS 6226, University of Rennes 1, Rennes Cedex, 35042, France
}

\begin{abstract}
Atomic-deficient void structure is studied in nanocomposites prepared by mechanochemical milling of glassy $\mathrm{g}-\mathrm{As}_{2} \mathrm{Se}_{3}$ in a water solution of polyvinylpyrrolidone (PVP) employing positron annihilation lifetime spectroscopy. Formalism of Ps-to-positron trapping conversion known as x3-x2-CDA (coupling decomposition algorithm) is applied to identify free-volume defects in the pelletized g-As $2 \mathrm{Se}_{3} / \mathrm{PVP}$ nanocomposite in respect to dry-milled g-As $\mathrm{Se}_{3}$ one. Under wet-milling, the inter-nanoparticle Ps-decaying sites in preferential PVP environment replace free-volume positron traps (in dry-milled g- $\mathrm{As}_{2} \mathrm{Se}_{3}$ ) with defect-specific lifetime of $0.352 \mathrm{~ns}$, corresponding to di-/tri-atomic vacancies in g-As-Se.
\end{abstract}

Keywords:, arsenic selenide, free-volume defect, nanoparticle, nanocomposite, positron annihilation, mechanical milling. PACS: 78.67.Sc, 78.70.Bj

\section{INTRODUCTION}

The chalcogenide compounds represented by $\mathrm{As}_{2} \mathrm{~S}_{3}$, different $\mathrm{As}_{4} \mathrm{~S}_{4}$ polymorphs, and $\mathrm{As}_{2} \mathrm{O}_{3}$, the overall known as arsenicals [1], have attracted a tight attention in scientific community because of their potential application as promising anti-cancer drugs in treatment of hematological malignant diseases [2]. Their medicinal activity can be tuned by nanostructurization stretching over atomic and sub-atomic length-scales [3-6] due to mechanochemistry, an advanced instrumentation tool ensuring arsenicals with unprecedented biocompatibility via enhanced ratio of surface-to-bulk states [7]. In medicinal implementations, the mechanically milled (MM) arsenicals are combined with organic stabilizers such as polyvinylpyrrolidone (PVP), to keep individuality of created nanoparticles (NPs), thereby producing PVP-capped arsenical nanocomposites [5,6,8-10]. Nowadays, there are no comparative information concerning similar functionalization in other types of arsenicals, such as arsenic selenides As-Se, forming a large group of glassy-like media possessing widespread application in IR photonics [11].

In this work, the free-volume structural arrangement in the PVP-encapsulated glassy $\mathrm{g}-\mathrm{As}_{2} \mathrm{Se}_{3}$ nanocomposites prepared by high-energy wet attrition ball milling is studied employing positron annihilation lifetime (PAL) spectroscopy, the non-destructive experimental probe for nanostructurization in composite materials $[10,12,13]$.

\section{EXPERIMENTAL}

\section{Nanocomposite preparation and characterization}

The studied nanocomposites were prepared using coarse-grained pieces of bulk g-As $\mathrm{Se}_{3}$ synthesized from highpurity commercial elemental precursors (As and $\mathrm{Se}$ of $5 \mathrm{~N}$ purity). The plane-parallel plates $(1.0-1.5 \mathrm{~mm}$ in a 
thickness) cut from thermally-annealed glassy ingots and polished to a high optical quality were used as reference samples for PAL spectroscopy measurement. The synthesized g-As $\mathrm{Se}_{3}$ has $4.590 \mathrm{~g} \cdot \mathrm{cm}^{-3}$ density determined at room temperature by the Archimedes displacement technique in ethanol, and mid-onset glass-transition temperature $\mathrm{T}_{\mathrm{g}}=185^{\circ} \mathrm{C}$ determined from DSC scans, as typical values proper to $\mathrm{g}-\mathrm{As}_{2} \mathrm{Se}_{3}[11]$.

The high-energy milling was performed in both dry and wet modes. The dry-mode milling was performed in Pulverissete 6 mill (operated at $500 \mathrm{rpm}$ rotational speed and protective argon atmosphere) for $20 \mathrm{~min}$ in $250 \mathrm{~mL}$ tungsten carbide chamber (loaded with 50 tungsten carbide balls each having $10 \mathrm{~mm}$ in diameter), using $3 \mathrm{~g}$ of coarse-grained $\mathrm{g}-\mathrm{As}_{2} \mathrm{Se}_{3}$ preliminary sieved under $200 \mu \mathrm{m}$. After milling, the powder was compressed by compacting inside a stainless steel die under a pressure of $\sim 0.7 \mathrm{GPa}$ to produce the dry-MM g- $\mathrm{As}_{2} \mathrm{Se}_{3}$ pellets having near $6 \mathrm{~mm}$ in a diameter and $1 \mathrm{~mm}$ in a thickness. The part of dry-milled powder was treated in a wet-milling mode. This 90-min attrition ball milling route performed in a presence of $300 \mathrm{~mL}$ of $0.5 \%$ PVP solution in laboratory MiniCer mill (Netzsch, Germany), operated under protective argon atmosphere at $3500 \mathrm{rpm}$ speed (85\% loading of milling shaft with yttrium-stabilized $\mathrm{ZrO}_{2}$ balls, each having $0.6 \mathrm{~mm}$ in a diameter). For this purpose, we used the powdered PVP with an average molecular weight of $40000 \mathrm{~g} \cdot \mathrm{mol}^{-1}$ purchased from Sigma-Aldrich Co. LLC (St. Louis, MO, USA). After filtration through sterile $0.22 \mu \mathrm{m}$ filter, the separated solid phase was dried at $70^{\circ} \mathrm{C}$ and pelletized under the same pressure of $\sim 0.7 \mathrm{GPa}$, thus producing the $\mathrm{g}-\mathrm{As}_{2} \mathrm{Se}_{3} / \mathrm{PVP}$ - nanocomposite.

The NPs sizes in milled arsenicals detected by photon cross-correlation spectroscopy reveal typical single modal distribution with $d_{50}=182 \mathrm{~nm}$ (i.e. $50 \%$ of NPs were smaller than $182 \mathrm{~nm}$ ) and $d_{99}=267 \mathrm{~nm}$ (i.e. $99 \%$ of NPs were smaller than $267 \mathrm{~nm}$ ). As it follows from X-ray diffractometry, the high-energy attrition ball milling in the above conditions does not change amorphous state of the substance.

\section{PAL spectra recording}

The PAL spectra were recorded with fast-fast coincidence system (ORTEC) of $230 \mathrm{ps}$ resolution stabilized at $22^{\circ} \mathrm{C}$ and relative humidity of $35 \%$. To ensure precise lifetime measurement, each PAL spectrum was recorded in normal-measurement statistics ( $\sim 10^{6}$ coincidences), the channel width of $6.15 \mathrm{ps}$ allowing 8000 channels in a total. The ${ }^{22} \mathrm{Na}$ isotope of $\sim 50 \mathrm{kBq}$ activity prepared from aqueous ${ }^{22} \mathrm{NaCl}$ solution (wrapped by Kapton ${ }^{\circledR}$ foil of $12 \mu \mathrm{m}$ thickness and sealed) was used as positron source sandwiched between two identical samples. The source correction ( $12 \%$ component contribution with 0.372 ns lifetime installed due to calibration with $\mathrm{Ni}$ and Kapton ${ }^{\circledR}$ foil) was applied to compensate input originating from annihilation in the source itself and in the covering Kapton ${ }^{\circledR}$ foil. The best fitting of the PAL spectra was achieved with LT 9.0 program [20] under decomposition into three single exponentials), covering channels from positrons annihilating in (i) defect-free bulky states, (ii) trapped in extended free-volume defects (positron-trapping sites) and (iii) forming bound positron-electron Ps (positronium) state. The error-bar in positron lifetimes $\tau_{\mathrm{i}}$ and intensities $\mathrm{I}_{\mathrm{i}}$ were $\pm 0.005 \mathrm{~ns}$ and $0.5 \%$, respectively. The PAL data were treated using two-state simple trapping model (STM), assuming one kind of free-volume defects for positrons $[12,15,16]$, disturbed by slight input from Ps-decaying. This approach allows determination of positron trapping modes (defectspecific $\tau_{d}$ and defect-free bulk $\tau_{b}$ lifetimes, trapping rate in defects $\kappa_{d}$, ) under condition of slight input from Psdecaying modes (i.e. radius of spherical holes $R$ and fractional free volume $f_{v}$ ).

\section{PAL spectra treatment with $x 3-x 2-C D A$}

For inhomogeneous solids, where annihilation is expected through mixed positron-Ps channels so that only Pstraps are transformed in positron-trapping centers (and vice versa), the formalism of Ps-to-positron trapping conversion $[5,6,13]$ can be applied to identify free-volume defects. Within this approach referred to as $x 3$-x2-CDA (coupling decomposition algorithm) [13], we deal with $\mathrm{x} 3$-term PAL spectrum transformed to generalized $\mathrm{x} 2$-term form for both reference and nanostructurized solids (caused, in part, by incorporation of guest NPs in host matrix), the second component involving all possible positron-trapping and Ps-decaying contributions. This approach allows resolving additional input with defect-specific $\tau_{\text {int }}$ lifetime and $\mathrm{I}_{\text {int }}$ intensity in the second component of reconstructed $\mathrm{x} 2$-term PAL spectrum, provided compensating $\left(\tau_{n}, \mathrm{I}_{\mathrm{n}}\right)$ input in the first component obeys complete equilibrium [13]. Parameterization of Ps-to-positron trapping conversion in the nanostructurized matrix is achieved accepting $\left(\tau_{n}, \mathrm{I}_{\mathrm{n}}\right)$ and $\left(\tau_{\text {int }}, I_{\text {int }}\right)$ as first and second components of $x 2$-term PAL spectrum employing two-state STM [12,15]. The trapping modes derived within this approach include defect-specific $\tau_{\text {int }}$ and defect-free bulk $\tau_{\mathrm{b}}{ }^{\mathrm{NP}}$ lifetimes, trapping rate in defects $\kappa_{d}{ }^{N P}$, signature of trap size in terms of equivalent number of vacancies defined by $\left(\tau_{\text {int }}-\tau_{b}{ }^{\mathrm{NP}}\right)$ difference and nature of these traps defined by $\tau_{\text {int }} / \tau_{\mathrm{b}}^{\mathrm{NP}}$ ratio [12]. 


\section{RESULTS AND DISCUSSION}

The PAL spectra of pelletized dry-MM and wet-MM g-As $\mathrm{Se}_{3}$, and bulk g-As ${ }_{2} \mathrm{Se}_{3}$ are built of three single exponentials (Table 1), the corresponding PAL trapping parameters being gathered in Table 2.

TABLE 1. PAL spectra fitting of $\mathrm{g}-\mathrm{As}_{2} \mathrm{Se}_{3}$-based arsenicals within free $\mathrm{x} 2$ - and $\mathrm{x} 3$-term decomposition.

\begin{tabular}{|c|c|c|c|c|c|c|c|}
\hline \multirow[t]{3}{*}{$\begin{array}{l}\begin{array}{l}\text { Arsenical sample } \\
\text { (fitting procedure) }\end{array} \\
\end{array}$} & \multirow{3}{*}{ [FIT-1] } & \multicolumn{5}{|c|}{ PAL spectra fitting parameters } & \multirow{3}{*}{$\begin{array}{l}\tau_{\text {av. }} \\
\text { ns }\end{array}$} \\
\hline & & $\tau_{1}$ & $\tau_{2}$ & $\tau_{3}$ & $\mathbf{I}_{2}$ & $\mathbf{I}_{3}$ & \\
\hline & & ns & ns & ns & a.u. & a.u. & \\
\hline Bulk g-Ass $\mathrm{Se}_{3}(\mathrm{x} 2$-term) & 0.07 & 0.209 & 0.363 & - & 0.627 & - & 0.305 \\
\hline Bulk g-Ass $\mathrm{Se}_{3}(\mathrm{x} 3$-term) & 0.01 & 0.193 & 0.358 & 2.091 & 0.620 & 0.007 & 0.310 \\
\hline Dry-MM g-As2 $\mathrm{Se}_{3}(\mathrm{x} 3$-term) & 0.05 & 0.202 & 0.371 & 2.087 & 0.540 & 0.015 & 0.322 \\
\hline Wet-MM g-Ass $\mathrm{Se}_{3}$ (x3-term) & 0.07 & 0.212 & 0.428 & 1.891 & 0.400 & 0.052 & 0.386 \\
\hline
\end{tabular}

The PAL spectrum of reference bulky g-As $\mathrm{Se}_{3}$ sample decomposed on three free exponentials reveals second component with $\tau_{2}$ lifetime approaching $\sim 0.36 \mathrm{~ns}$, which is at a character level of triple- and quadruple-type vacancies in As-Se matrix [17-19]. The third component with relatively small fractional free volume $\mathrm{f}_{\mathrm{v}}=0.15$ and $\tau_{3}=2.091 \mathrm{~ns}$ can be ascribed to Ps-related holes having a radius of $0.296 \mathrm{~nm}$. Despite small number of corresponding Ps-decaying sites, this component cannot be eliminated from the PAL spectrum without notable reduction in fitting goodness. Nevertheless, we also decomposed the measured PAL spectrum of bulky g- $\mathrm{As}_{2} \mathrm{Se}_{3}$ in evidently worse x2term form (due to seven-times increased [FIT-1] value), to compare these results with known data for the same glass treated within two-state STM $[12,15]$. The good coincidence in defect-specific $\tau_{\mathrm{d}}$ and defect-free bulk $\tau_{\mathrm{b}}$ lifetimes, as well as trapping rate in defects $\kappa_{d}$ speaks in a favor of tight proximity between these reference samples.

TABLE 2. PAL trapping modes of $g$ - $\mathrm{As}_{2} \mathrm{Se}_{3}$-based arsenicals within free $\mathrm{x} 2$ - and $\mathrm{x} 3$-term decomposition

\begin{tabular}{|c|c|c|c|c|c|c|c|c|}
\hline \multirow[t]{2}{*}{ Arsenical sample } & \multicolumn{6}{|c|}{ Positron-trapping modes } & \multicolumn{2}{|c|}{ Ps-decay modes } \\
\hline & $\tau_{\text {av. }}{ }^{\text {tr }}$ & $\tau_{\mathbf{b}}$ & $\kappa_{d}$ & $\tau_{2}-\tau_{b}$ & $\tau_{2} / \tau_{\mathrm{b}}$ & $\eta$ & $\mathbf{R}_{3}$ & $\mathbf{f}_{\mathrm{v}}{ }^{3}$ \\
\hline & ns & ns & $\mathrm{ns}^{-1}$ & ns & a.u. & a.u. & $\mathrm{nm}$ & $\%$ \\
\hline Bulk g-As $2 \mathrm{Se}_{3}(\mathrm{x} 2$-term) & 0.305 & 0.285 & 1.27 & 0.08 & 1.27 & 0.27 & - & - \\
\hline Bulk g-Ass2 $\mathrm{Se}_{3}(\mathrm{x} 3$-term) & 0.297 & 0.272 & 1.51 & 0.09 & 1.32 & 0.29 & 0.296 & 0.15 \\
\hline Dry-MM g-As $2 \mathrm{Se}_{3}(\mathrm{x} 3$-term $)$ & 0.295 & 0.270 & 1.24 & 0.10 & 1.37 & 0.25 & 0.296 & 0.29 \\
\hline Wet-MM g-Ass $\mathrm{Se}_{3}(\mathrm{x} 3$-term) & 0.304 & 0.270 & 1.01 & 0.16 & 1.58 & 0.21 & 0.277 & 0.83 \\
\hline
\end{tabular}

In structurally inhomogeneous substances composed of agglomerated NPs having individual 100-300 nm grains (as it clearly illustrated by particle size distribution on Fig. 1), the large fraction of positrons thermalized inside these grains travelers towards grain boundaries (because of their diffusion length becomes comparable with NP size), where they annihilate after getting trapped into free-volume defects. This feature determines a more complicated PAL spectroscopy for both dry-MM and wet-MM g-As $\mathrm{Se}_{3}$ (as compared with non-milled bulky samples) in addition to large amount of free-volume voids and unfilled cavities created due to powdering these arsenicals [20].

The x3-term decomposed PAL spectrum of the pelletized dry-MM g-As ${ }_{2} \mathrm{Se}_{3}$ demonstrates slightly increased defect-specific lifetime $\tau_{2}$, but rather unchanged Ps-decaying lifetime $\tau_{3}$, the average lifetime $\tau_{\text {av. as a mass center of }}$ all positron-annihilation events [12] being only slightly shifted from 0.310 ns to 0.322 ns. The third component intensity $\mathrm{I}_{3}$ is over two-times increased as in bulky g- $\mathrm{As}_{2} \mathrm{Se}_{3}$, resulting in equivalent growth of fractional free volume

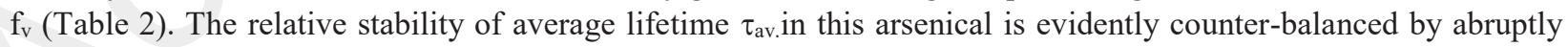
decreased second component intensity $\mathrm{I}_{2}$, the process favoring changes in the Ps-formation probability [21].

The x3-term PAL spectrum of the pelletized PVP-capped g- $\mathrm{As}_{2} \mathrm{Se}_{3}$ nanocomposite (i.e. wet-MM g- $\mathrm{As}_{2} \mathrm{Se}_{3}$ ) differ essentially demonstrating obvious changes in positron- and Ps-related annihilation channels, the corresponding changes in third and second component intensities $\left(I_{2}\right.$ and $\left.I_{3}\right)$ being enhanced evidently (Table 1$)$. As a consequence, the average lifetime $\tau_{\mathrm{av}}$ in this composite grows to $0.386 \mathrm{~ns}$, while the $\tau_{3}$ lifetime drops to $1.891 \mathrm{~ns}$, tending towards Ps-related lifetime in pure PVP matrix ( $\tau_{3}=1.867 \mathrm{~ns}$ ) [22]. Hence, the Ps-decaying occurs rather in PVP environment than in "pure" g-As-Se matrix, this process being nearly three-times enhanced in respect to $\mathrm{f}_{\mathrm{v}}=0.83 \%$ (see Table 2). In contrast, the defect-specific $\tau_{2}$ lifetime grows abnormally in $\mathrm{g}-\mathrm{As}_{2} \mathrm{Se}_{3} / \mathrm{PVP}$ nanocomposite approaching $\sim 0.43 \mathrm{~ns}$, which can be ascribed to positron trapping in large free-volume defects such as multi-vacancy clusters [17-19]. 
To identify free-volume nanostructurization in the pelletized $\mathrm{g}-\mathrm{As}_{2} \mathrm{Se}_{3} / \mathrm{PVP}$ nanocomposite, the PAL spectrum of pelletized dry-milled $\mathrm{g}-\mathrm{As}_{2} \mathrm{Se}_{3}$ is considered in respect to wet-milled one using the $\mathrm{x} 3$-x2-CDA [13] (Table 3).

TABLE 3. $x 3-x 2-C D A$ trapping modes in dry-MM g-As $2 \mathrm{Se}_{3}$ defined in respect to g-As2 $\mathrm{Se}_{3} / \mathrm{PVP}$ nanocomposite.

\begin{tabular}{|c|c|c|c|c|c|c|c|c|c|c|}
\hline \multicolumn{3}{|c|}{ First component } & \multicolumn{3}{|c|}{ Second component } & \multicolumn{5}{|c|}{ Positron trapping modes } \\
\hline$\tau_{\mathrm{n}}$ & $\mathbf{I}_{\mathbf{n}}$ & $\left(\tau_{n} \cdot I_{n}\right)$ & $\tau_{\text {int }}$ & I int & $\left(\tau_{\text {int }} \cdot \mathbf{I}_{\text {int }}\right)$ & $\tau_{\mathrm{av}} \mathrm{NP}$ & $\tau_{b}{ }^{N P}$ & $\kappa_{d}{ }^{N P}$ & $\tau_{\text {int }-\tau_{b}}{ }^{N P}$ & $\tau_{\text {int }} / \tau_{\mathrm{b}} \mathrm{NP}$ \\
\hline ns & a.u. & ns & ns & a.u. & ns & ns & ns & $\mathrm{ns}^{-1}$ & ns & a.u. \\
\hline 0.194 & 0.334 & 0.065 & 0.352 & 0.472 & 0.166 & 0.287 & 0.263 & 1.35 & 0.09 & 1.34 \\
\hline
\end{tabular}

The positive first and second component intensities ( $\mathrm{I}_{\mathrm{n}}$ and $\mathrm{I}_{\mathrm{int}}$ ) in the PAL spectrum reconstructed in terms of $\mathrm{x} 3-\mathrm{x} 2-\mathrm{CDA}$, along with well-filled $\left(\tau_{\mathrm{n}} \cdot \mathrm{I}_{\mathrm{n}}\right)$ and $\left(\tau_{\mathrm{int}} \cdot \mathrm{I}_{\mathrm{int}}\right)$ component inputs testify that Ps-decaying sites in pelletized PVP-g- $\mathrm{As}_{2} \mathrm{Se}_{3}$ nanocomposite are transformed to positron-trapping sites in dry-MM g- $\mathrm{As}_{2} \mathrm{Se}_{3}$ pellets. The defectrelated lifetime $\tau_{\text {int }}=0.352$ ns shows the appeared positron traps represent interfacial voids, which are close in their free volumes to di- and/or tri-atomic vacancies in g-As-Se matrix, reaching $\sim 0.25-0.28 \mathrm{~nm}$ in a radius (in a spherical approximation) as it follows from known semiempirical relationships for arsenic triselenide $\mathrm{As}_{3} \mathrm{Se}_{3}$ [17-19]. This finding agrees well with $\left(\tau_{\mathrm{int}}-\tau_{\mathrm{b}}{ }^{\mathrm{NP}}\right)=0.09 \mathrm{~ns}$ and $\tau_{\text {int }} / \tau_{\mathrm{b}}{ }^{\mathrm{NP}}=1.34$ values as character indicatives of these vacancies [12]. In dry-MM g- $\mathrm{As}_{2} \mathrm{Se}_{3}$ pellet, this positron-trapping channel substitutes the Ps-decaying contribution originated from inter-NPs holes with $\tau_{3}=2.087 \mathrm{~ns}$ by input from PVP-filled inter-NPs Ps-traps with $\tau_{3}=1.891 \mathrm{~ns}$ lifetime (Table 1). The whole trapping-conversion process, i.e. appearance of positron traps with defect-related lifetime $\tau_{\text {int }}=0.352 \mathrm{~ns}$ instead of Ps-decaying sites with $\tau_{3}=2.087 \mathrm{~ns}$ lifetime, occurs in a chemical environment possessing defect-free bulk positron lifetime $\tau_{b}{ }^{\mathrm{NP}}=0.263 \mathrm{~ns}$ (Table 3 ). This value is evidently above bulk positron lifetime in crystalline $\mathrm{c}-\mathrm{As}_{2} \mathrm{Se}_{3}$ $\left(\tau_{\mathrm{b}}=0.240 \mathrm{~ns}\right)$ [17], but below this lifetime in $\mathrm{g}-\mathrm{As}_{2} \mathrm{Se}_{3}\left(\tau_{\mathrm{b}}=0.280-0.285 \mathrm{~ns}\right)$ as defined within STM from constrainedfree x2-term decomposed PAL spectra, i.e. ignoring any competitive contribution from Ps-decaying channel [19]. Thus, the chemical environment of Ps-to-positron trapping conversion, i.e. inner spaces where positron traps appear (dry-MM g-As2 $\mathrm{Se}_{3}$ ) instead of PVP-filled inter-NPs Ps-decay sites (wet-MM g-As $\mathrm{Se}_{3}$ ), is indeed g-As-Se matrix.

In our consideratio, we have been grounded on assumption that Ps-to-positron trapping conversion is alone process linking dry-MM and wet-MM g-As $\mathrm{Se}_{3}$. This condition seems to be really satisfied under small amount of PVP in nanocomposite due to the same positron annihilation channel from defect-free bulky states in both arsenicals.

\section{REFERENCES}

1. P. J. Dilda and P. J. Hogg, Cancer Treatment Rev. 33 542-564 (2007).

2. W. Ding, L. Zhang, S. Kim, W. Tian, Y. Tong, J. Liu, Y. Ma and S. Chen, Molecular Medicine Rep. $11968-974$ (2015).

3. Y. Tian, X. Wang, R. Xi, W. Pan, S. Jiang, Z. Li, Y. Zhao, G. Gao and D. Liu, Intern. J. Nanomedicine $9745-757$ (2014).

4. J.-Z. Wu and P.C. Ho, Eur. J. Pharm. Sci. 29 35-44 (2006).

5. Z. Bujnakova, P. Balaz, P. Makreski, G. Jovanovski, M. Caplovicova, L. Caplovic, O. Shpotyuk, A. Ingram, T.-C. Lee, J.-J. Cheng, J. Sedlak, E. Turianicova and A. Zorkovska, J. Mater. Sci. 50 1973-1985 (2015).

6. O. Shpotyuk, Z. Bujňáková, P. Baláž, A. Ingram, P. Demchenko, A. Kovalskiy, M. Vlcek, Ya. Shpotyuk, J. Cebulski and A. Dziedzic, Mater. Chem. Phys. 186 251-260 (2017).

7. P. Baláž, M. Achimovičová, Z. Bujňáková and E. Dutková, J. Mater. Sci. 52 11851-11890 (2017).

8. G. Ghosh, M.K. Naskar, A. Patra and M. Chatterjee, Opt. Mater. 28 1047-1053 (2006).

9. I. Saravanan, S. Diwakar, R. Mohankumar, A. Pandurangan and R. Jayavel, Nanomater. Nanotechnol. 1 $42-48$ (2011).

10. O. Shpotyuk, A. Ingram, Y. Shpotyuk, Z. Bujňáková and P. Baláž, Polymer Eng. Sci. 57 502-505 (2017).

11. A. Feltz, Amorphous Inorganic Materials and Glasses, VCH, Weinheim, 1993, pp. 1-446.

12. R. Krause-Rehberg and H. Leipner, Positron annihilation in semiconductors: defect studies, Heidelberg, Springer, 1999 , pp. $1-350$.

13. O. Shpotyuk, J. Filipecki, A. Ingram, R. Golovchak, M. Vakiv, H. Klym, V. Balitska, M. Shpotyuk and A. Kozdras, Nanoscale Res. Lett. 10 77-1-77-5 (2015).

14. J. Kansy, Nucl. Instr. Meth. Phys. Res. A 374 235-244 (1996).

15. F. Tuomisto and I. Makkonen, Rev. Mod. Phys. 85 1583-1631 (2013).

16. Y.C. Jean, P.E. Mallon and D.M. Schrader, Principles and application of positron and positronium chemistry. World Sci. Publ. Co. Pte. Ltd., New Jersy-London-Singapore-Hong Kong, 2003.

17. K.O. Jensen, P.S. Salmon, I.T. Penfold and P.G. Coleman, J. Non-Cryst. Solids 170 57-64 (1994).

18. O. Shpotyuk, A. Ingram, M. Shpotyuk and J. Filipecki, Nucl. Instr. Meth. Phys. Res. B 338 66-71 (2014).

19. M. Hyla, J. Filipecki, O. Shpotyuk, M. Popescu and V. Balitska, J. Optoelectron. Adv. Mat. 9 3177-3181 (2007).

20. O. Shpotyuk, A. Ingram, Z. Bujňáková, P. Baláž and Ya. Shpotyuk, Nanoscale Res. Lett. 11 10-1-10-7 (2016).

21. Y.K. Vijay, S. Wate, D.K. Awasthi, D. Das and S. Ghughre, Indian J. Eng. Mater. Sci. 7 375-377 (2000).

22. O. Shpotyuk, Z. Bujňáková, P. Baláž, A. Ingram and Ya. Shpotyuk, J. Pharm. Biomed. Anal. 117 419-425 (2016). 